\title{
GENETIC EVOLUTION OF HUMAN CORONAVIRUS OC43 IN NEURAL CELL CULTURE
}

\author{
Julien R. St-Jean, Marc Desforges, and Pierre J. Talbot*
}

\section{INTRODUCTION}

Human coronaviruses $(\mathrm{HCoV})$ are ubiquitous in the environment and are responsible for up to one-third of common colds. $\mathrm{HCoV}-\mathrm{OC} 43$ possesses a genome that comprises genes encoding various structural and nonstructural proteins. Amongst these proteins, the $\mathrm{S}$ protein is biologically very important because it could be involved in determination of viral tropism. Indeed, it could for instance be associated with the capacity of the virus to reach the central nervous system (CNS) and possibly trigger neurological disorders. It could also confer the host species specificity observed with coronaviruses. In past years, we have shown that HCoV-OC43 is neurotropic and neuroinvasive, as it persistently infects neural cell cultures ${ }^{1}$ and human brains. ${ }^{2}$ Although we have suggested that OC43 could remain genetically surprisingly stable in the environment, ${ }^{3}$ it is known that coronaviruses can adapt in cell culture or under selection pressure, for instance related to immune system evasion.

\section{MATERIALS AND METHODS}

\subsection{Viruses, Cell Lines, and Persistent Infections}

The ATCC HCoV-OC43 strain (VR-759) was grown on the HRT-18 rectal tumor cell line. Persistent infection were carried out in those HRT-18 cells, as well as in the MO3.13 oligodendrocytic, ${ }^{4}$ H4 neuroglial, U-87 MG astrocytic, and TE-671 rhabdomyosarcoma cell lines (ATCC). Other cell lines used for virus susceptibility are described in Table 2. Four infections were performed in the H4 cell line, whereas the HRT-18, MO3.13, and H4 cell lines were acutely infected as controls.

*INRS-Institut Armand-Frappier, Laval, Québec, H7V 1B7 Canada. 


\subsection{Virus Purification}

Virus from persistent infections was purified at different passages. Prior to purification, virus was clarified and precipitated with polyethylene glycol (PEG) 8000 (Sigma). Accudenz (Accurate Chemicals) was used to perform gradient purification.

\subsection{RT-PCR and Sequencing}

Viral RNA was extracted using the GenElute Direct mRNA Miniprep Kit (Sigma) and reverse transcribed with MMuLVreverse transcriptase (Invitrogen). The Expand High-Fidelity Taq polymerase (Roche) was used to perform PCR. Primers specific to the $\mathrm{HE}, \mathrm{S}$ and $\mathrm{N}$ genes were used to amplify target regions. ${ }^{3} \mathrm{PCR}$ amplicons were purified using the Qiaex II gel extraction kit (Qiagen) prior to sequencing, which was carried out by Bio S\&T (Montréal, Québec, Canada).

\subsection{Assays for Viral Susceptibillity and Modulation of Tropism and Infectivity}

Prior to performing assays for modulation of tropism and infectivity, susceptibility of different cell lines to HCoV-OC43, ATCC strain, was determined (Table 2). The same cell lines were then infected with virus isolated from different purifications (HRT-18 P33, P54, P110, and P155; H4 P47 and P90; H4 P56.1, P56.2, P56.3, P116.1, P116.2, and P116.3; TE-671 P38 and P79; U-87 MG P35, and MO3.13 P5, P6, and P22) in order to correlate the observed mutations with a modulation of tropism or infectivity. Supernatants were titrated using an indirect immunoperoxidase assay (IPA), as previously described. ${ }^{5}$

\section{RESULTS}

Persistent infections of neural cell lines were initially performed to determine whether virus carrying mutations in genes encoding the surface protein $\mathrm{S}$ originated as a consequence of viral persistence. The HE protein gene and the nucleocapsid protein gene $\mathrm{N}$ were also sequenced in order to determine if these genes contributed to adaptation in cell culture. Viral particles released from persistently infected neural cell lines were isolated and purified by gradient centrifugation, and genomic RNA was sequenced. Results showed various mutations in the $\mathrm{S}$ gene but very few in $\mathrm{HE}$ and $\mathrm{N}$ genes, suggesting that the $\mathrm{S}$ gene is responsible for adaptation to the cellular environment, which could be associated with neurotropism, neuroinvasion, and presumably neuropathogenesis (Table 1). Almost every acquired mutation (Table 1) was conserved at subsequent passages, suggesting that they could confer an adaptive advantage and a stable phenotype to the virus. Five mutations were predominant and were found in almost all persistent infections (D24Y, S83T, H183R, Y241H, and N489H). The first four mutations are located in the putative receptor binding site, whereas the fifth one is located within the hypervariable region.

To correlate the observed mutations in the $\mathrm{S}$ gene with viral replication and tropism, assays for modulation of tropism and infectivity ${ }^{6}$ were performed using cell lines originating from various human tissues as well as from various animal species, for which 
Table 1. Location of $\mathrm{S}$ mutations at various passages of persistently infected cells.

\begin{tabular}{|c|c|c|c|c|c|c|c|}
\hline HRT-18 & H4 & H4 & H4 & H4 & TE-671 & U-87 M & 03.13 \\
\hline P155** & P90 & P116.1 & P116.2 & P116.3 & P79 & P35 & P22 \\
\hline D30H* & D24Y & $\mathrm{D} 24 \mathrm{Y}$ & D24Y & $\mathrm{N} 25 \mathrm{Y}$ & $\mathrm{N} 27 \mathrm{Y}$ & D24Y & D115H \\
\hline S83T & V161V & P35S & P35S & P35S & P34S & S83T & T148I \\
\hline L85Q & H183R & S83T & S147Y (D) & S83T & L85R & H183R & Y241H \\
\hline D115H & V240V & E170K & H183R & Y119H & S258R & $\mathrm{Y} 241 \mathrm{H}$ & M670T \\
\hline T148I & Y241H & H183R & Y241H & S147P & A373V & & P973S \\
\hline H183Q & N441K & Y241H & N441K & H183R & R757S & & A1090V \\
\hline S258R & Q541L & $\mathrm{A} 469 \mathrm{~V}$ & E460D & Y241H & G785D & & V1213A \\
\hline S366G & R570P & R570R & $\mathrm{H} 482 \mathrm{Y}$ & $\mathrm{N} 489 \mathrm{H}$ & P972L & & \\
\hline N413T & N639N & T855I & F683Y & K506T & P973S & & \\
\hline F420S & T855I & N880K (I) & L693F & $\mathrm{T} 641 \mathrm{~S}$ & A978S & & \\
\hline N489H & D875H & L893H & A759E & N768T & $\mathrm{T} 1086 \mathrm{~N}$ & & \\
\hline K506N & L893R & S959C & S898S (I) & E896K & D1170A & & \\
\hline T536N & A965V & W974L & V980A & S901F & & & \\
\hline Q541L & T975A & T975P & N1203 (D) & W974L & & & \\
\hline R757H & I1227T & V980A & I1227T & F982L & & & \\
\hline E896D & T1245I & S1093S & & V986I & & & \\
\hline C897G & & G1169G & & G1169D & & & \\
\hline E933G & & M1222K & & E1236A & & & \\
\hline F982L & & D1232Y & & & & & \\
\hline S1192R & & P1249L & & & & & \\
\hline T1225I & & I1304I & & & & & \\
\hline P1228S & & & & & & & \\
\hline
\end{tabular}

susceptibility to HCoV-OC43 infection was previously determined (Table 2). These analyses revealed that mutations found throughout the $\mathrm{S}$ gene could affect the latter viral properties in certain cell lines. Amongst the virus variants obtained following persistent infections and virus purifications, five showed extended cellular tropism and increased replication titers in vitro: U87-MG P35, H4 P47, H4 P56.3, H4 P116.1, and H4 P116.2 (data not shown). Furthermore, some variants isolated from persistent infections were more virulent in mice and could form plaques, in opposition to the ATCC HCoV-OC43 reference strain VR759 (data not shown).

\section{DISCUSSION}

We have identified several mutations in the $\mathrm{S}$ gene of the HCoV-OC43 genome following persistent infections in different cell lines. These mutations will help us to further characterize viral adaptation during persistence and to understand mechanisms that are implicated in viral tropism and infectivity. Future studies will be carried out using an infectious cDNA clone of the OC43 strain assembled in a BAC vector. ${ }^{7,8}$ The construction of this clone was performed in collaboration with F. Almazán and L. Enjuanes and will provide an invaluable tool to further understanding the underlying mechanisms for viral replication and tropism. In combination with the experiments described above, the clone will be useful in elucidating the molecular basis of human coronavirus neuropathogenesis. 
Table 2. Susceptibility of various cell lines to the HCoV-OC43 ATCC strain.

\begin{tabular}{|c|c|c|c|c|c|}
\hline Cell line & Origin & Tissue & Type & IPA $^{1}$ & Susceptibility $^{2}$ \\
\hline HeLa & Human & Uterus & Epithelial & 3.75 & Low \\
\hline MT4 & Human & Bone marrow & T lymphocyte & 2.0 & No \\
\hline U937 & Human & Bone marrow & Monocyte & $\leq 0.5$ & No \\
\hline Jurkat E6.1 & Human & Bone marrow & T lymphocyte & $\leq 1.5$ & No \\
\hline Raji & Human & Bone marrow & B lymphocyte & 4.5 & Yes \\
\hline HL-60 & Human & Bone marrow & Monocyte & 3.25 & Low \\
\hline WI-38 & Human & Lung & Fibroblast & 4.0 & Yes \\
\hline L132 & Human & Lung & Epithelial & 3.25 & Low \\
\hline Caki-2 & Human & Kidney & Epithelial & $\leq 1,5$ & No \\
\hline SW 156 & Human & Kidney & Epithelial & $\leq 1.5$ & No \\
\hline NCI-N87 & Human & Stomach & Epithelial & $\leq 1.75$ & No \\
\hline Arpe-19 & Human & Eye & Epithelial & $\leq 1.5$ & No \\
\hline FHs 74 Int & Human & Intestine & Epithelial & 3.5 & Low \\
\hline TK6 & Human & Spleen & T lymphocyte & $\leq 1.75$ & No \\
\hline $17 \mathrm{Cl}-1$ & Mouse & Embryo & Fibroblast & $\leq 1.75$ & No \\
\hline L929 & Mouse & Subcutaneous & Fibroblast & $\leq 1.5$ & No \\
\hline N-11 & Mouse & Brain & Microglial & $\leq 1.5$ & No \\
\hline DBT & Mouse & Brain & Glial & $\leq 2.25$ & No \\
\hline J774 A.1 & Mouse & Bone marrow & Macrophage & 4.5 & Yes \\
\hline A20 & Mouse & Bone marrow & B lymphocyte & 3.25 & Low \\
\hline S.END.1 & Mouse & Skin & Endothelial & $\leq 1.5$ & No \\
\hline Cos -7 & Monkey & Kidney & Fibroblast & $\leq 1.5$ & No \\
\hline Vero & Monkey & Kidney & Epithelial & $\leq 1.75$ & No \\
\hline Vero E.6 & Monkey & Kidney & Epithelial & $\leq 1.5$ & No \\
\hline B104 & Rat & Brain & Fibroblast & 3.25 & Low \\
\hline BHK-21 & Hamster & Kidney & Fibroblast & 4.0 & Yes \\
\hline
\end{tabular}

${ }^{1}$ Indirect immunoperoxidase assay (infectious titers in $\mathrm{TCID}_{50} / \mathrm{mL}$ ).

${ }^{2}$ Titers from 0 to 3 , not susceptible; titers over 3 and under 4 , low susceptibility; titers of 4 and over, susceptible.

\section{REFERENCES}

1. Arbour, N., Côté, G., Lachance, C., Tardieu, M., Cashman, N. R., and Talbot, P. J., 1999, Acute and persistent infection of human neural cell lines by human coronavirus OC43, J. Virol. 73:3338-3350.

2. Arbour, N., Day, R., Newcombe, J., and Talbot, P. J., 2000, Neuroinvasion by human respiratory coronaviruses, J. Virol. 74:8913-8921.

3. St-Jean, J. R., Jacomy, H., Desforges, M., Vabret, A., Freymuth, F., and Talbot, P. J., 2004, Human respiratory coronavirus OC43: Genetic stability and neuroinvasion, J. Virol. 78:8824-8834.

4. McLaurin, J., Trudel, G. C., Shaw, I. T., Antel, J. P., and Cashman, N. R., 1995, A human glial hybrid cell line differentially expressing genes subserving oligodendrocyte and astrocyte phenotype, J. Neurobiol. 26:183-193.

5. Jacomy, H., and Talbot, P. J., 2003, Vacuolating encephalitis in mice infected by human coronavirus OC43, Virology 315:20-33.

6. Schickli, J. H., Zelus, B. D., Wentworth, D. E., Sawicki, S. G., and Holmes, K. V., 1997, The murine coronavirus mouse hepatitis virus strain A59 from persistently infected murine cells exhibits an extended host range, J. Virol. 71:9499-9507.

7. Almazán, F., Gonzalez, J. M., Pénzes, Z., Izeta, A., Calvo, E., Plana-Duran, J., and Enjuanes, L., 2000, Engineering the largest RNA virus genome as an infectious bacterial artificial chromosome, Proc. Natl. Acad. Sci. USA 97:5516-5521.

8. St-Jean, J. R., Desforges, M., Almazán, F., Jacomy, H., Eujuanes, L. and Talbot, P. J., 2006. Recovery of a neuro virulent human coronavirus OC43 from an infectious cDNA clone. J. Virol. 80:3670-3674. 